Proc. 21st Winter Workshop on

Nuclear Dynamics (2005) 000-000

21st Winter Workshop on Nuclear Dynamics

Breckenridge, Colorado, USA

February 5-12, 2005

\title{
Direct Photon Measurement at RHIC-PHENIX
}

\author{
Takao Sakaguchi for the PHENIX Collaboration \\ Brookhaven National Laboratory, \\ Physics Department, Upton, NY 11973, U.S.A.
}

\begin{abstract}
Results on direct photon measurements from the PHENIX experiment at RHIC are presented. The direct photon yields for $p_{T}>6 \mathrm{GeV} / c$ as a function of centrality in $\mathrm{Au}-\mathrm{Au}$ collisions at $\sqrt{s_{N N}}=200 \mathrm{GeV}$ are found to be consistent with NLO pQCD calculation scaled by the number of binary collisions. The results suggest that the photons observed are emitted from the initial stage of hard scattering. Comparisons with several theoretical calculations are also presented.
\end{abstract}

Keywords: Direct Photons, Au, RHIC, PHENIX, QGP, pQCD

PACS: 12.38.Mh, 12.38.-t, 24.85.+p, 25.75.Nq

\section{Introduction}

The Relativistic Heavy Ion Collider (RHIC) at BNL is expected to produce a hot and dense matter, whose energy density is sufficiently high to induce a phase transition to the so-called Quark Gluon Plasma (QGP) [1]. RHIC has been operated for five years since the first collision of $\mathrm{Au}+\mathrm{Au}$ in 2000, and produced many intriguing results that have never appeared in lower center-of-mass energy systems [ 2]. Especially, the energy loss of hard-scattered parton in the hot and dense medium that results in "jet quenching" has been of great interest since its first discovery by the PHENIX experiment [3]. The hard scattered probe now become an useful tool to explore system dynamics because its cross-section is large at RHIC energy, and can be accurately calculated by perturbative QCD (pQCD). The results obtained so far including jet quenching suggest that a hot dense medium has been created.

Photons are excellent electromagnetic probes for extracting the thermodynamical information of where they are emitted because they do not interact strongly with medium once produced. Thus, they are expected to provide hints to answer questions of whether the jet quenching is due to initial or final state effect, and if the medium turned into the QGP. The photon production processes are shown in Fig. [1 The Compton scattering of quarks and gluons and the annihilation of quarks and 
(a)

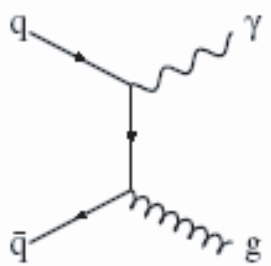

Annihilation

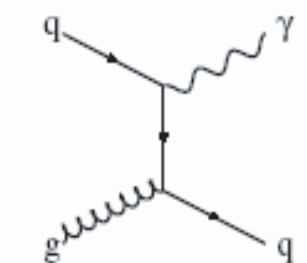

Compton Scattering (b)

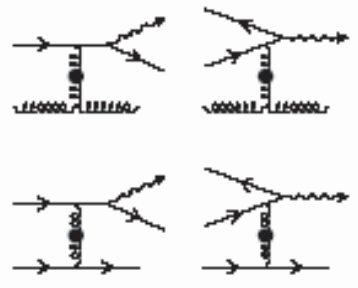

Bremsstrahlung

Fig. 1. Photon production in (a) leading order process, and (b) next-to-leading order process.

anti-quarks are leading order processes, and the next leading process is dominated by bremsstrahlung. There is also a prediction of a jet-photon conversion process, which occurs if QGP is formed, by a secondary interaction of a hard scattered parton with thermal partons in the medium [4]. Fig. 2] shows a theoretical prediction of photons over a $p_{T}$ range [5]. The calculation predicts that a photon contribution

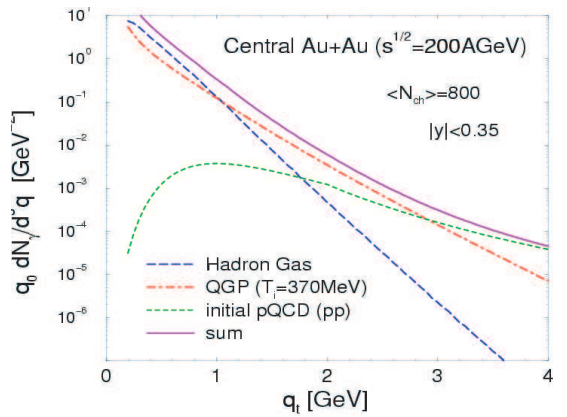

Fig. 2. Theoretical calculation on various photon contributions for central $\mathrm{Au}+\mathrm{Au}$ collisions at $\sqrt{s_{N N}}=200 \mathrm{GeV}$.

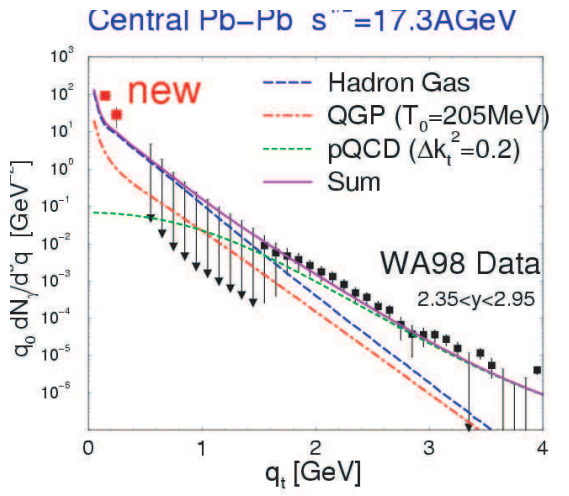

Fig. 3. WA98 results and their theoretical interpretation.

from the QGP state is predominant in the $p_{T}$ range of $1<p_{T}<3 \mathrm{GeV} / c$. The signal can be seen after subtracting photons decaying from known hadronic sources. The typical signal to background ratio is $\sim 10 \%$ [ [6]. For $p_{T}>3 \mathrm{GeV} / c$, the signal is dominated by a contribution from initial hard scattering, and $p_{T}<1 \mathrm{GeV}$, the signal is from hadron gas through processes of $\pi \pi(\rho) \rightarrow \gamma \rho(\pi), \pi K^{*} \rightarrow K \gamma$ and etc..

Direct photon measurements in heavy ion collisions were first carried out by the WA80 experiment in 200A GeV S+Au collisions, where only the upper limits are obtained [ 7]. The WA98 experiments observed a significant excess in the $p_{T}$ region of $1<p_{T}<3 \mathrm{GeV} / c$ in $158 \mathrm{~A} \mathrm{GeV} \mathrm{Pb}+\mathrm{Pb}$ collisions [ [8, 9], and a recent theoretical calculation interpreted it as a consequence of either $k_{T}$ broadening or high initial temperature [10] as shown in Fig. 3] Since there is no direct photon result for $\mathrm{p}+\mathrm{Pb}$ collisions, one can not evaluate the effect of $k_{T}$ broadening in $\mathrm{Pb}+\mathrm{Pb}$ collisions. 
The E706 Collaboration at Fermilab measured direct photons in $\pi+\mathrm{Be}, \mathrm{p}+\mathrm{Be}$ at 515 and $800 \mathrm{GeV} / c$, and showed that the data deviate from NLO pQCD calculation without including additional $k_{T}$ smearing according to the incident particle and target species [11]. The result suggests that the Cronin-like enhancement can be explained by the initial $k_{T}$ broadening. The STAR experiment at RHIC measured inclusive photons in the $p_{T}$ region of $0.5<p_{T}<2.5 \mathrm{GeV} / c$, and compared it to the possible decay $\gamma$ contributions from $\pi^{0}$ [12]. Since the systematic error is order of $40-50 \%$ in the measurement, it is not possible to conclude whether there is a significant excess or not.

In this paper, the latest results on direct photon measurement from the PHENIX experiment are shown, and the source of the photon contribution is discussed.

\section{PHENIX detector}

Figure 4 shows the PHENIX detector at RHIC. Both central arms (East and West)

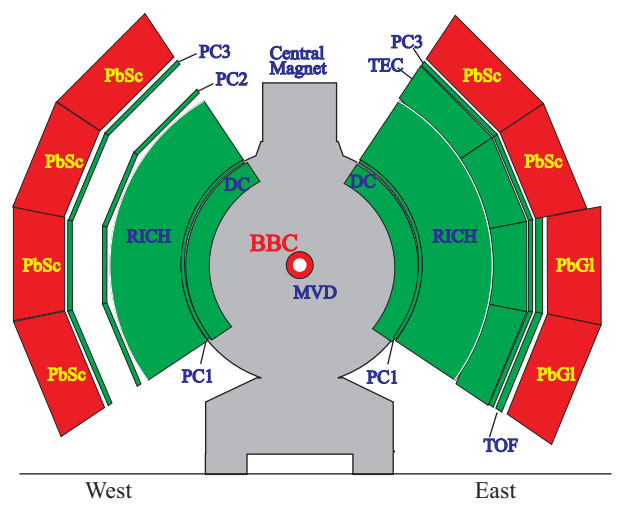

Fig. 4. The PHENIX detector.

of the detector include a Multiplicity Vertex Detector (MVD), Drift Chamber (DC), Pad Chamber (PC), Ring Imaging Cerenkov Counter (RICH) and Electro-Magnetic Calorimeter (EMCal), and cover a rapidity range of $|\eta|<0.35$ and a quarter azimuth. The East arm also has a Time Of Flight (TOF) detector and Time Expansion Chamber (TEC). The detailed description of the detector is given in the literature [13]. In this analysis, EMCal was used for measuring the energy of photons. The EMCal consists of six lead-scintillator sandwich type calorimeters $(\mathrm{PbSc})$ and two leadglass homogeneous type calorimeters $(\mathrm{PbGl})$. The $\mathrm{DC}$ and $\mathrm{PC}$ were used to track charged particles to estimate charged hadron contamination in EMCal clusters.

The analysis of direct photons require determining background photons decaying from known hadronic sources such as $\pi^{0}$ and $\eta$. PHENIX has measured the transverse momentum spectra of $\pi^{0}$ up to $13 \mathrm{GeV}$ in $\mathrm{Au}+\mathrm{Au}$ collisions [ 14, 15] and $\mathrm{p}+\mathrm{p}$ collision [16] at $\sqrt{s_{N N}}=200 \mathrm{GeV}$. The momentum spectra of $\eta$ and other hadronic sources were estimated by using a fit to $\pi^{0} p_{T}$ spectrum and applying $m_{T}$ scaling: $p_{T} \rightarrow\left(p_{T}^{2}-M_{\pi}^{2}+M_{h}^{2}\right)^{1 / 2} . \eta$ has been measured but only $\eta / \pi^{0}$, which 


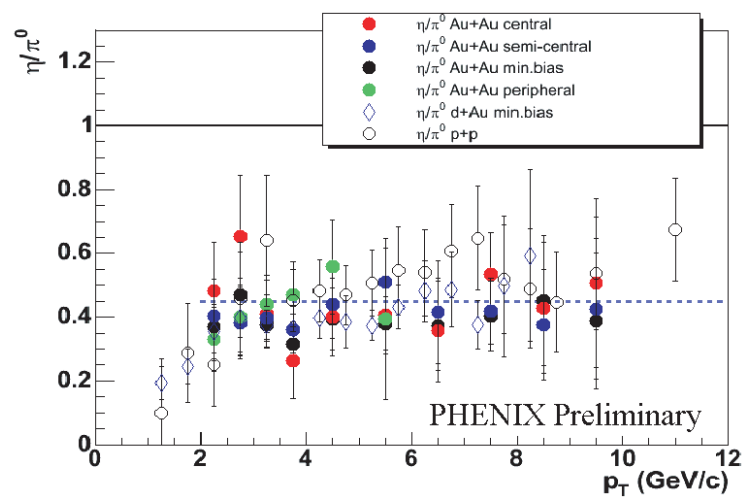

Fig. 5. $\eta$ to $\pi^{0}$ ratio for $\mathrm{p}+\mathrm{p}, \mathrm{d}+\mathrm{Au}$ and $\mathrm{Au}+\mathrm{Au}$ collisions at $\sqrt{s_{N N}}=200 \mathrm{GeV}$. The average value of $0.45 \pm 0.05$ is obtained.

is $0.45 \pm 0.05$ as seen in Fig. [5 was used to determine the normalization factor of the $m_{T}$-scaled spectra relative to $\pi^{0}$. Using above fits, the number and spectra of photons decaying from $\pi^{0}$ and $\eta$ are obtained.

The inclusive photon spectra were reconstructed by applying several photon ID cuts on the measured cluster energy distributions, and correcting for hadron contamination and PID efficiency. In order to identify photons, a PID likelihood function is calculated from several quantities measured by EMCal such as shower shape or ratio of energies among towers, and apply a threshold. It resulted in a significant reduction of hadronic clusters in the sample. Since the ratio of $\gamma$ to $\pi^{0}$ cancels their common systematic errors, the excess of the measured photon over the estimated background photon is evaluated in terms of $R_{\gamma} \equiv\left(\gamma / \pi^{0}\right)_{\text {measured }} /\left(\gamma / \pi^{0}\right)_{\text {estimated }}$ (double ratio). The final systematic error on the double ratio is $\sim 12-16 \%$, and $\sim 15-20 \%$ on direct photon spectra in $\mathrm{Au}+\mathrm{Au}$ collisions [17].

\section{Results}

Fig. 6] shows the direct photon spectra in $\mathrm{p}+\mathrm{p}$ collisions together with the NLO pQCD calculation with three different cut-off scales [18]. The statistical error is shown as error bars, and the systematic error as boxes. The data are well described by the calculation. Fig. 7 shows a compilation of comparisons of data to NLO pQCD calculations in $\mathrm{p}+\mathrm{p}$ and $\mathrm{p}+\mathrm{A}$ collisions [19]. As are most of the data points in the figure, the PHENIX $\mathrm{p}+\mathrm{p}$ data points shown in red are consistent with $\mathrm{pQCD}$ expectation within a factor of 2 . The result shows that the NLO pQCD calculation describes well the yield of direct photons from the initial hard scattering process in $\mathrm{p}+\mathrm{p}$ collisions at $\sqrt{s}=200 \mathrm{GeV}$. Fig. 8 shows the $\gamma / \pi^{0}$ double ratio over various centralities in $\mathrm{Au}+\mathrm{Au}$ collisions together with NLO pQCD calculation scaled by the number of binary collisions in red lines. The shaded bands on the data points show the systematic errors, and the bars are the statistical errors. The shaded bands around the lines show the uncertainty of the pQCD calculation. The magnitude 


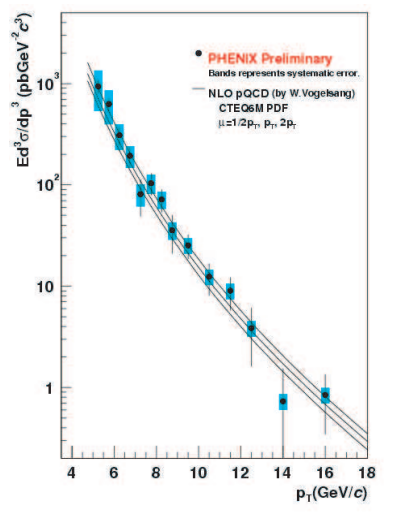

Fig. 6. Direct Photon spectrum in $\mathrm{p}+\mathrm{p}$ collisions at $\sqrt{s}=200 \mathrm{GeV}$.

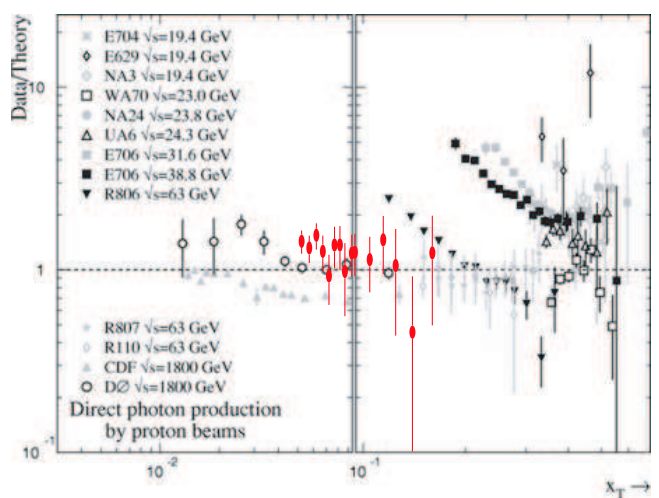

Fig. 7. Compilation of comparisons of data to NLO pQCD calculations. PHENIX data are shown as red points.

of the excess at and above $p_{T} \sim 4 \mathrm{GeV} / c$ increases with increasing centrality of collisions, and is consistent with the calculation. Fig. 9 shows the direct photon spectra extracted as $\gamma_{\text {direct }}=\left(1-R_{\gamma}{ }^{-1}\right) \cdot \gamma_{\text {measured }}$. The lines show the same NLO

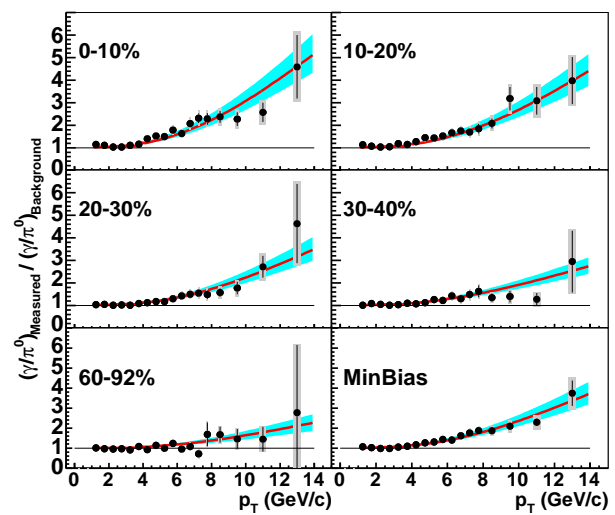

Fig. 8. $\gamma / \pi^{0}$ double ratio in $\mathrm{Au}+\mathrm{Au}$ collisions.

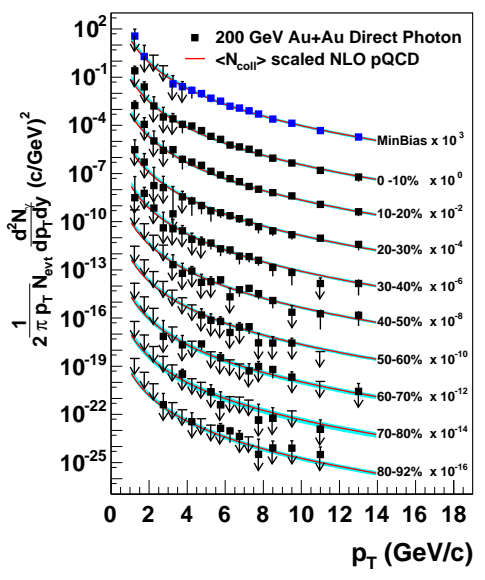

Fig. 9. Direct photon spectra in $\mathrm{Au}+\mathrm{Au}$ collisions over centralities.

pQCD calculation scaled by the number of binary collisions. It is clearly seen that all nine centralities including minimum bias are well described by the calculation. Considering the fact that the calculation in Fig. 8 took the suppression of $\pi^{0}$ into account which results in less background photons in higher centralities, and that the Fig. 9 shows the good agreement between the data and the calculation, we conclude that the suppression of $\pi^{0}$ is not attributed to the initial hard scattering process, while the direct photon is. Fig. 10 shows nuclear modification factor $R_{A A}$ 
as a function of centrality, represented by $N_{\text {part }}$, which is defined by:

$$
R_{A A}=\frac{\left(1 / N_{A A}{ }^{e v t}\right) d^{2} N_{A A} / d p_{T} d y}{<N_{\text {coll }}>/ \sigma_{p p}^{\text {inel }} \cdot d^{s} \sigma_{p p} / d p_{T} d y} .
$$

The dotted lines show the uncertainty on the number of binary collisions, and the

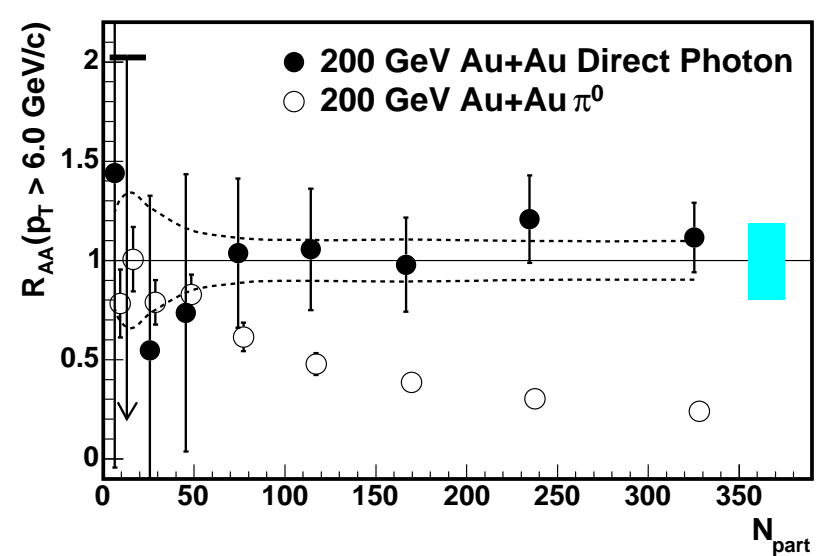

Fig. 10. Nuclear Modification Factor $\left(R_{A A}\right)$ for integrated yield of $p_{T}>6 \mathrm{GeV}$ for $\pi^{0}$ (open circles) and direct photons (solid circles).

boxes shows the uncertainty of the NLO pQCD calculation. The sold circle points are for $\pi^{0}$ [ 14], which clearly shows a suppression with increasing centrality. In case of the direct photons shown in open circles, the yield follows the number of binary collisions scaling. It once again proved that the suppression of $\pi^{0}$ is final state effect.

\section{Discussion}

There have been several theoretical calculations on photons from various contributions. Fig. [11] shows the comparison of 0-10\% central PHENIX data with three NLO pQCD calculations [20, 21], two of which have same formulation with different $k_{T}$ smearing values, and with the calculation including jet-photon conversion process [ 4. The NLO pQCD calculations with different $k_{T}$ smearing values show a difference in the yields below $p_{T} \sim 6 \mathrm{GeV} / c$, where the data points have large error and can not distinguish between models. The difference of yield decreases as $p_{T}$ increases because of less steep slope at higher $p_{T}$. The model including jet-photon conversion describes the data very well for $p_{T}>4 \mathrm{GeV} / c$. Fig. 12 shows the comparison of the same data with the model with jet-photon conversion to its components. It can be seen that the jet-photon conversion process dominates a $p_{T}$ region of $4-7 \mathrm{GeV} / c$, therefore, it should dominate the integrated yields for $p_{T}>6 \mathrm{GeV} / c$. Accordingly, this yield should follow the number binary collisions scaling. Thus, the contribution from jet-photon conversion process should scale with the number of collisions 


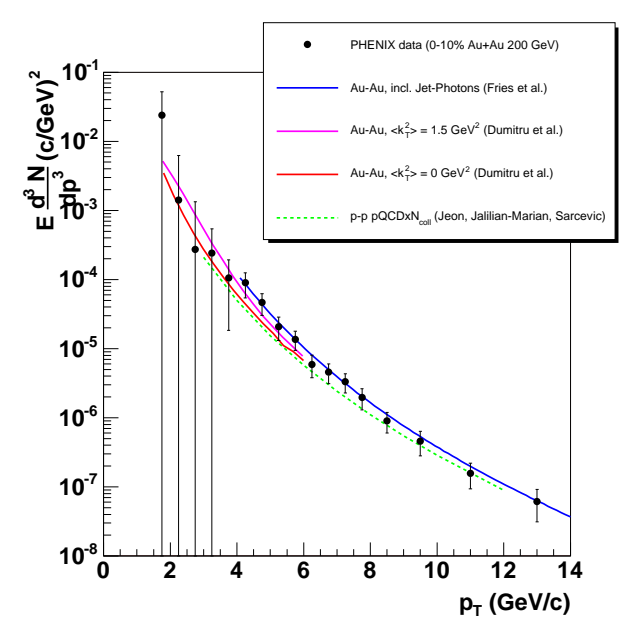

Fig. 11. Comparison of $0-10 \%$ central PHENIX data with NLO pQCD calculations and jet-photon conversion included model.

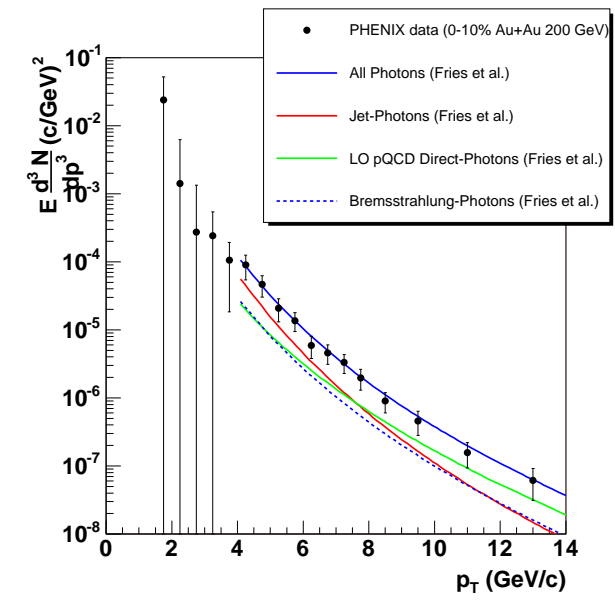

Fig. 12. Comparison of the same data with the model with jet-photon conversion to its components.

as well. However, the process assumes the existence of a hot and dense medium or QGP, which is not the case in peripheral collisions. The model does not give consistent description of collisions for all centralities. The data are also compared with calculation on Fig. [6] and shown on Fig. 13 Within the current error, the

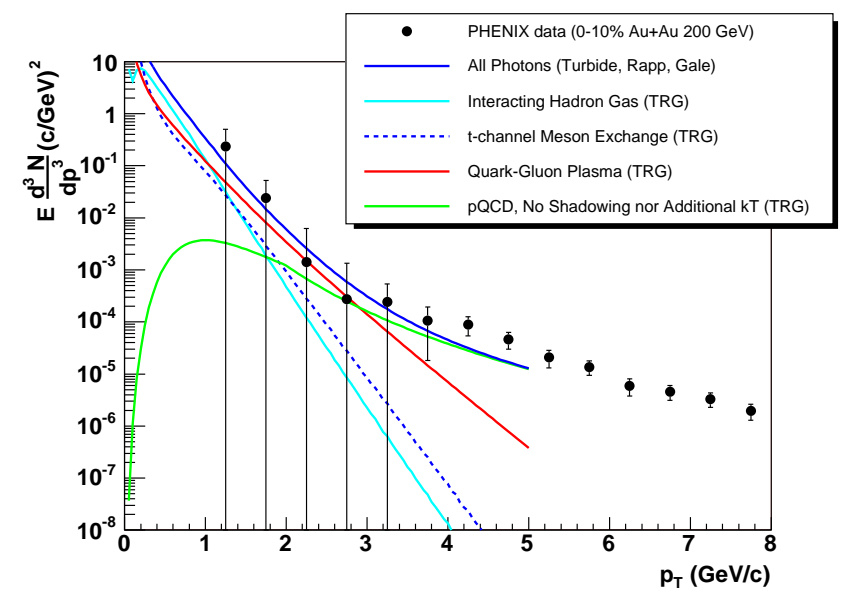

Fig. 13. Comparison of the same data with the calculation shown in Fig. [6

calculation is consistent with the data up to $4 \mathrm{GeV} / c$, and starts deviating from the data above $4 \mathrm{GeV} / c$. However, It is possible that the fact that pQCD calculation in the plot does not include an additional $k_{T}$ smearing can in part explain the deviation. 


\section{Conclusion}

Results on direct photon measurements from the PHENIX experiment at RHIC are presented. The direct photon yields for $p_{T}>6 \mathrm{GeV} / c$ in $\mathrm{Au}-\mathrm{Au}$ collisions at $\sqrt{s_{N N}}=200 \mathrm{GeV}$ are found to be consistent with NLO pQCD calculation scaled by the number of binary collisions. The results suggest that the photons observed are emitted from the initial stage of hard scattering. Comparisons with several theoretical calculations are also presented.

The direct photon analysis in $\mathrm{d}+\mathrm{Au}$ collisions and high statistics $\mathrm{Au}+\mathrm{Au}$ collisions with the goal to reduce systematics error and disentangle the contributions from QGP state is now ongoing.

\section{References}

1. T.D. Lee, Rev. Mod. Phys. 47(1975)267.

2. See for example, K. Adcox et al. (PHENIX Collaboration), nucl-ex/0410003

3. K. Adcox et al. (PHENIX Coll.), Phys. Rev. Lett. 88, 022301 (2002).

4. R.J. Fries, B. Müller and D.K. Srivastava, Phys. Rev. Lett. 90, 132301 (2003).

5. S. Turbide, R. Rapp and C. Gale, Phys. Rev. C69, 140903 (2004).

6. F. Arleco et al., hep-ph/0311131

7. R. Albrecht et al. (WA80 Coll.) Phys. Rev. Lett.76, 3506 (1996).

8. M.M. Aggarwal et al. (WA98 Coll.), Phys. Rev. Lett. 85, 3595 (2000).

9. M.M. Aggarwal et al. (WA98 Coll.), Phys. Rev. Lett. 93, 022301 (2004).

10. R. Rapp, Mod. Phys. Lett. A19 (2004) 1717.

11. L. Apanasevich et al. (Fermilab E706 Coll.), Phys. Rev. D70, 092009 (2004).

12. C. Adler et al. (STAR Coll.), Phys. Rev. C70, 044902 (2004).

13. K. Adcox et al. (PHENIX Coll.), Nucl. Inst. \& Meth. A499 (2003) 469.

14. S.S. Adler et al. (PHENIX Coll.), Phys. Rev. Lett. 91, 072301 (2003).

15. J. Frantz (PHENIX Coll.), J. Phys. G30 (2003) S1003.

16. S.S. Adler et al. (PHENIX Coll.), Phys. Rev. Lett. 91, 241803 (2003).

17. S.S. Adler et al. (PHENIX Coll.), nucl-ex/0503003

18. L.E. Gordon and W. Vogelsang, Phys. Rev. D48, 3136 (1993).

19. L. Apanasevich et al., Phys. Rev. D63, 014009 (2000).

20. A. Dmitriu et al., Phys. Rev. C64, 054909 (2002).

21. S. Jeon, J. Jalilian-Marian and I. Sarcevic, Nucl. Phys. A715 (2003) 795. 